\title{
Exploring the mechanism of WWOX growth inhibitory effects on oral squamous cell carcinoma
}

\author{
WEI YANG $^{1 *}$, XIAO-MING WANG ${ }^{2 *}$, HONG-YAN YUAN $^{3}$, ZHI-HUI LIU ${ }^{4}$, SHUANG GAO ${ }^{1}$ and LIANG PENG ${ }^{1}$ \\ ${ }^{1}$ School of Laboratory Medicine, Beihua University, Jilin City, Jilin 132013; ${ }^{2}$ Department of Pathology, \\ Jilin Province People's Hospital; ${ }^{3}$ Department of Immunology, College of Basic Medical Science, Jilin University, \\ Changchun, Jilin 132001; ${ }^{4}$ Department of Stomatology, Taizhou Municipal Hospital, Taizhou, Zhejiang 318000, P.R. China
}

Received August 15, 2015; Accepted January 17, 2017

DOI: $10.3892 / \mathrm{ol} .2017 .5850$

\begin{abstract}
Oral squamous cell carcinoma (OSCC) is one of the most common types of head and neck neoplasms in the world. Patients diagnosed with OSCC exhibit a poor prognosis. WW domain-containing oxidoreductase (WWOX), as a candidate tumor-suppressor gene, is involved in the genesis and progression of tumors. The deletion of the WWOX gene has been identified in OSCC and oral leukoplakia, but the function and mechanism of WWOX in OSCC remain unknown. Therefore, the present study investigated the role of WWOX in oral squamous carcinoma cells. The results revealed that an elevation of WWOX expression had an inhibitory effect on the growth of three types of oral squamous carcinoma cells, with the most evident effect occurring in Tca8113 cells. Also, in the Tca8113 cells, WWOX overexpression significantly inhibited colony formation, and induced apoptosis and cell cycle arrest. Microarray analysis, reverse transcription-quantitative polymerase chain reaction and western blotting methods detected that WWOX overexpression contributed to the differential expression of the genes involved in mediating the extracellular-signal regulated protein kinase/mitogen-activated protein kinase (ERK/MAPK) signaling pathway. These results suggest that the tumor-suppressor function of the WWOX gene may be associated with the modulation of the ERK/MAPK signaling pathway, thus providing a novel target for OSCC therapy.
\end{abstract}

\section{Introduction}

Each year, 480,000 new cases of oral cancer are diagnosed worldwide, the majority of which are squamous cell

Correspondence to: Dr Zhi-Hui Liu, Department of Stomatology, Taizhou Municipal Hospital, 381 Zhong Shan East Road, Taizhou, Zhejiang 318000, P.R. China

E-mail: liuzhihui1209@sina.com

*Contributed equally

Key words: WW domain-containing oxidoreductase, tumorsuppressor gene, oral squamous cell carcinoma, mitogen-activated protein kinase, extracellular-signal regulated protein kinase carcinoma $(1,2)$. Globally, oral squamous cell carcinoma (OSCC) has become the most commonly diagnosed type of head and neck neoplasm (3). Despite improvements in tumor treatments, the 5-year survival rate for OSCC has remained $\sim 50 \%$ during the last decades (4). In the treatment of OSCC, surgery and radiation can cause severe functional impairment, while the efficacy of chemotherapeutic agents is limited by acquired resistance and drug side effects (5). As a result, the development of new molecular targets for the prevention and treatment of OSCC has become an urgent matter.

WW domain-containing oxidoreductase (WWOX) has been identified as a tumor-suppressor gene that spans the chromosomal fragile site FRA16D. Lost or reduced expression of the WWOX gene commonly presents in numerous types of neoplasms, including breast, prostate, ovary, lung and oral cancer (6-8). A reduced expression of WWOX has been observed in cases of OSCC and oral leukoplakia, which are prevalent precancerous lesions $(8,9)$. By contrast, normal transcriptomes and proteins of WWOX were observed to be expressed in normal mucosa $(9,10)$. Therefore, the present study speculated that WWOX deficiency in oral squamous carcinoma cells may contribute to oral carcinogenesis. However, the function and precise molecular mechanism of WWOX in OSCC remain unclear.

The present study aimed to detect, in vitro, the effect of WWOX overexpression on cell growth, apoptosis and cell cycle distribution in oral squamous carcinoma cells. The present study also used a microarray assay, reverse transcription-quantitative polymerase chain reaction (RT-qPCR) analysis and western blotting to investigate the expression of genes that are modulated by WWOX, in order to elucidate the underlying molecular mechanisms of the antitumor effect of WWOX.

\section{Materials and methods}

Cell lines and cell culture. The oral cancer cell lines, Tca8113, CAL27 and FaDu, were provided by Dr Zhiyong Li (Stomatology Hospital Affiliated to Zhejiang University College of Medicine, Hangzhou, China). These cells and 293T cells [Type Culture Collection of Chinese Academy of Sciences (TCCCAS), Beijing, China] were cultured in Dulbecco's modified Eagle's medium (Gibco; Thermo Fisher Scientific, Inc., 
Waltham, MA, USA) supplemented with $10 \%$ fetal bovine serum (GE Healthcare Life Sciences, Chalfont, UK). The cells were grown in an incubator at $37^{\circ} \mathrm{C}$ with a humidified atmosphere containing $5 \% \mathrm{CO}_{2}$.

Plasmid construction, virus packaging and cell transfection. The WWOX gene, NM_016373, open reading frame was amplified from human breast carcinoma MCF-7 cells (TCCCAS, Beijing, China) using the following specific primers; forward, 5'-GAGGATCCCCGGGTACCGGTCGCC ACCATGGCAGCGCTGCGCTAC-3' and reverse, 5'-TCC TTGTAGTCCATACCGCCGGACTGGCTGCCAAG-3'. The WWOX complementary DNA (cDNA) was subcloned into a pGV287-LV lentivirus vector, purchased from Shanghai Genechem Co., Ltd. (Shanghai, China). The positive clone, pGV287-LV-WWOX, was selected through sequencing. The reconstructed lentivirus vector, and two helper vectors, pHelper1.0 and pHelper2.0 (Jikai Gene Biochemical Co., Ltd., Shanghai, China), were produced in the 293T packaging cell line. The cells were placed in 6-well plates and grown to $30 \%$ confluency prior to being infected with the virus $(\sim 109 \mathrm{TU} / \mathrm{ml}$, multiplicity of infection $=20$ ). The medium was replaced at $16 \mathrm{~h}$ post-infection and the cells were cultured as normal. RT-qPCR and western blotting were then used to confirm the expression of WWOX, according to the protocol described below.

Cell proliferation assay. An MTT assay was used to assess cell proliferation. The Tca8113 cells, the Tca8113 cells transfected with an empty vector and the Tca8113 cells transfected with the reconstructed vector were separately plated in 96-well plates with a density of 2,000 cells/well and incubated at $37^{\circ} \mathrm{C}$ for 1-5 days. A total of $20 \mu 1 \mathrm{MTT}(5 \mathrm{mg} / \mathrm{ml})$ was added to each well and incubated for $4 \mathrm{~h}$ on days 1 to 5 . Each day, prior to incubation, a total of $20 \mu \mathrm{l}$ MTT $(5 \mathrm{mg} / \mathrm{ml})$ was added to each well and the plates were incubated for $4 \mathrm{~h}$. The crystals formed were subsequently dissolved in dimethyl sulfoxide. The optical density was assessed at $490 \mathrm{~nm}\left(\mathrm{OD}_{490}\right)$. Cell proliferation activity was analyzed using the mean $\mathrm{OD}_{490}$ values for each well.

Colony formation assay. Subsequent to infection, the cells were seeded in 6-well plates at a density of 800 cells/plate and cultured at $37^{\circ} \mathrm{C}$ for 14 days until visible colonies appeared. The cells were stained with methyl violet prior to end of the incubation and the number of colonies was counted under a light microscope (Olympus Corporation, Tokyo, Japan).

Apoptosis and cell cycle analysis. The cells were harvested, then washed and resuspended in PBS $72 \mathrm{~h}$ following transfection, then stained with propidium iodide for cell cycle analysis. Apoptosis analysis was performed using an Annexin V-APC Apoptosis Detection kit (eBioscience, Inc., San Diego, CA, USA), according to the protocol of the manufacturer.

Microarray analysis. Total RNA from six samples, consisting of three samples from Tca8113 cells with WWOX overexpression and three samples from Tca8113 cells infected with the empty lentivirus vector, was isolated. TRIzol reagent (Invitrogen; Thermo Fisher Scientific, Inc.) was used to lyse the cells, then chloroform and isopropanol were used to isolate the RNA from the cell lysate, and alcohol treated with diethyl pyrocarbonate was used to inactivate RNases. The RNA samples were tested using NanoDrop 2000 (Thermo Fisher Scientific, Inc., Wilmington, DE, USA) and a 2100 Bioanalyzer (Agilent Technologies, Inc., Santa Clara, CA, USA). The RNA samples that met the following criteria: NanoDrop 2000, 1.7< Absorbance $(\mathrm{A})_{260} / \mathrm{A}_{280}<2.2$; and 2100 Bioanalyzer, RNA integrity number $\geq 7.0$ and $28 \mathrm{~S} / 18 \mathrm{~S}>0.7$, were analyzed by microarray expression profiling using GeneChip ${ }^{\circledR}$ PrimeView $^{\mathrm{TM}}$ Human Gene Expression Array (Affymetrix, Inc., Santa Clara, CA, USA) according to standard protocol (11). Genes that were significantly differentially expressed in cells, with or without WWOX overexpression, were selected based on exhibiting $\mathrm{P}<0.05$ and a 1.5 -fold-change (FC). The Reactome FI Cytoscape Plugin (www.reactome.org) was used to perform function pathway analysis according to the protocol of the manufacturer.

$R T-q P C R$. Each reaction was carried out in triplicate. Total RNA separately from the Tca8113 cells with empty vectors and the Tca8113 cells from with WWOX overexpression was isolated from the cultured cells using TRIzol reagent (Invitrogen; Thermo Fisher Scientific, Inc.) for cDNA synthesis. Reverse transcription was performed for one cycle under the following conditions: $70^{\circ} \mathrm{C}$ for $10 \mathrm{~min} ; 42^{\circ} \mathrm{C}$ for $1 \mathrm{~h}$; then $70^{\circ} \mathrm{C}$ for $10 \mathrm{mi} ß \mathrm{n}$ using the Reverse Transcription System (Promega Corporation, Madison, WI, USA). qPCR was performed using a StepOneä Real-Time PCR System (Applied Biosystems, Carlsbad, CA, USA) with the One Step SYBR PrimeScript RT-PCR kit II (Takara Bio, Inc., Otsu, Japan). The relative expression of all investigated genes was calculated using the $2^{-\triangle \Delta C q}(12)$ method subsequent to the normalization of the reference gene GAPDH. The primer sequences were as follows: GAPDH forward, 5'-TGACTTCAACAGCGACAC CCA-3' and reverse, 5'-CACCCTGTTGCTGTAGCCAAA-3'; WWOX forward, 5'-CCAACCACCCGGCAAAGATA-3' and reverse, 5'-AATGCTGCACGCTACGGAG-3'; dual specificity phosphatase (DUSP) 5 forward, 5'-TCCTCACCTCGCTAC TCG-3' and reverse, 5'-ACATCCACGCAACACTCAG-3'; DUSP6 forward, 5'-GAACTGTGGTGTCTTGGTACATT-3' and reverse, 5'-GTTCATCGACAGATTGAGCTTCT-3'; nuclear receptor subfamily 4 group A member 1 (NR4A1) forward, 5'-TCATGGACGGCTACACAG-3' and reverse, 5'-GTGGCTGAGGACGAGGATG-3'; mitogen-activated protein kinase kinase (MAP2 K) 5 forward, 5'-ACTGACGAG CGGTGGAC-3' and reverse, 5'-GTCGGAAGGTTCTGGA-3'; and fibroblast growth factor receptor 2 (FGFR2) forward, 5'-GGTGGCTGAAAAACGGGAAG-3' and reverse, 5'-AGA TGGGACCACACTTTCCATA-3'. PCR was performed under the following conditions: $95^{\circ} \mathrm{C}$ for $30 \mathrm{sec} ; 40$ cycles of $95^{\circ} \mathrm{C}$ for $5 \mathrm{sec}$ and $60^{\circ} \mathrm{C}$ for $30 \mathrm{sec}$.

Western blot analysis. Cell proteins were separated using $10 \%$ SDS-PAGE and transferred to a polyvinylidene fluoride membrane subsequent to cell protein concentration being quantified with a Bio-Rad assay kit (cat. no. 5000002; Bio-Rad Laboratories, Inc., Hercules, CA, USA). The membranes were blocked in 5\% non-fat milk at room temperature for $1 \mathrm{~h}$ and incubated with the primary antibodies (all antibodies were purchased from Santa Cruz Biotechnology, Inc., Dallas, 
TX, USA) anti-WWOX (dilution, 1:200; cat. no. sc-390175), anti-DUSP5 (dilution, 1:500; cat. no. sc-393801), anti-DUSP6 (dilution, 1:500; cat. no. sc-137245), anti-NR4A1 (dilution, 1:500; cat. no. sc-365113), anti-MAP2K5 (dilution, 1:500; cat. no. sc-135986) and anti-FGFR2 (dilution, 1:500; cat. no. sc-6930), at $4^{\circ} \mathrm{C}$ overnight. Subsequent to 3 washes in TBS/ $0.1 \%$ Tween-20, the membranes underwent hybridization with a peroxidase-conjugated secondary antibody (dilution, 1:2,000; cat. no. sc-2031; Santa Cruz Biotechnology, Inc.) for $1 \mathrm{~h}$ at room temperature. Signals were then visualized with the BeyoECL Plus detection kit (Beyotime Institute of Biotechnology, Haimen, China).

Statistical analysis. All quantitative data were presented as the mean \pm standard deviation. One-way analysis of variance and Student's t-test were used to compare the normally distributed continuous variables. The statistical significance of the microarray results was analyzed using FC values and a Student's t-test. $\mathrm{P}<0.05$ was considered to indicate a statistically significant difference. Statistical analysis was performed with SPSS 16.0 (SPSS, Inc., Chicago, IL, USA).

\section{Results}

Lentivirus infection enables the overexpression of the WWOX gene in Tca8113 cells. By comparing the relative WWOX messenger RNA (mRNA) levels in the oral cancer Tca8113, CAL27 and FaDu cell lines, the present study observed that Tca8113 cells exhibited the lowest expression of the WWOX gene $(\mathrm{P}<0.05$; Fig. 1A). Subsequent to the Tca8113 cells being infected with the lentivirus plasmid pGV287-LV-WWOX, the mRNA expression of the WWOX gene was $>90.593$-fold, significantly greater than that detected in the cells infected with the empty vector pGV287-LV ( $\mathrm{P}<0.05$; Fig. 1B). Western blotting detected elevated levels of WWOX protein expression in Tca8113 cells with WWOX overexpression (Fig. 1C).

Overexpression of the WWOX gene inhibits cell growth in vitro. To investigate the effect of WWOX on cell growth, WWOX overexpression plasmids were transfected into Tca8113, CAL27 and FaDu cells. WWOX overexpression inhibited cell proliferation as determined by the MTT method. This inhibition was most prevalent in the Tca8113 cells $(\mathrm{P}<0.05$; Fig. $2 \mathrm{~A}$ and $\mathrm{B})$. The colony formation assay also revealed similar inhibition in the Tca8113 cells $(\mathrm{P}<0.05$; Fig. 2C). Furthermore, flow cytometry assays revealed a significant increase in apoptosis and G0/G1-phase population subsequent to transfection with the WWOX overexpression plasmids ( $\mathrm{P}<0.05$; Fig. 2D and $\mathrm{E})$.

Overexpression of the WWOX gene modulates several key pathways in Tca8113 cells, as determined by global gene expression analysis. To investigate the mechanism of WWOX tumor-suppressor function, the present study compared the transcriptomes of cells transfected with the WWOX gene with those transfected with an empty vector. The present study used the Affimetrix Human Gene 1.0 ST Array to identify 347 transcriptomes that were significantly differentially expressed, of which, 171 genes were upregulated and 176 genes were downregulated, based on an FC $>1.5$ and $\mathrm{P}<0.05$ threshold
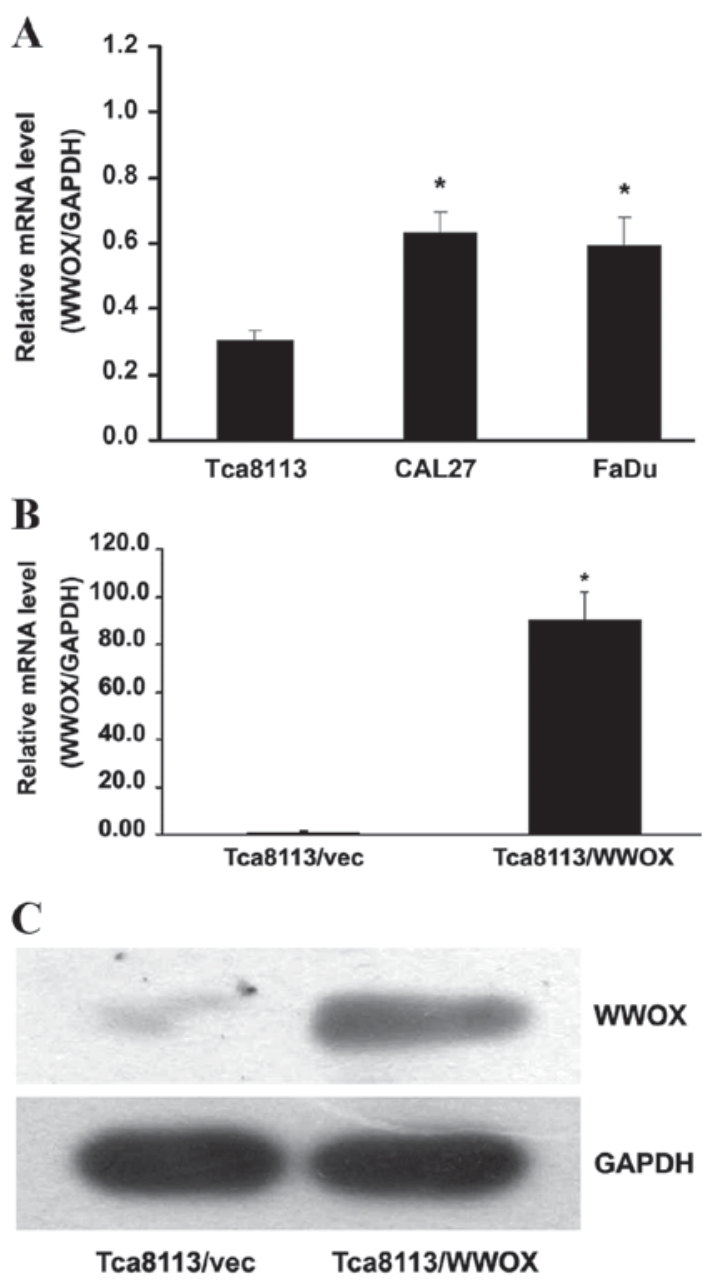

Figure 1. WWOX expression is elevated in Tca8113 cells. (A) RT-qPCR demonstrating that the Tca8113 cells exhibited the lowest expression of WWOX among the three types of oral cancer cells evaluated. ${ }^{*} \mathrm{P}<0.05$ compared with the Tca8113 cells. (B) WWOX mRNA was upregulated in the Tca8113/WWOX group. The results were detected by RT-qPCR and calculated as previously described. " $\mathrm{P}<0.05$ compared with the Tca8113/vec group. (C) WWOX protein expression level in the Tca8113/WWOX group, as assessed by western blotting. WWOX, WW domain-containing oxidoreductase; vec, empty vector; RT-qPCR, reverse transcription-quantitative polymerase chain reaction; mRNA, messenger RNA.

in Tca8113 cells with WWOX overexpression compared with the findings in the associated control (Fig. 3A). Through a functional analysis of genes using the Reactome Functional Interaction network, the present study demonstrated that WWOX overexpression modulated the activation of certain key signaling pathways, including the mitogen-activated protein kinase (MAPK) signaling pathway (Fig. 3B and C).

Overexpression of the WWOX gene regulates the expression of certain genes involved in modulating MAPK signaling. The present study screened selected genes associated with MAPK signaling. Utilizing global gene expression analysis to determine absolute $\mathrm{FC}$ subsequent to the overexpression of WWOX, the present study identified that the absolute FC of DUSP5, DUSP6, NR4A1 and MAP2K5 increased, while the absolute FC of FGFR2 decreased, compared with that of the Tca8113/vector control group (all $\mathrm{P}<0.05$; Fig. 4A). Subsequently, the mRNA and protein expression levels of 

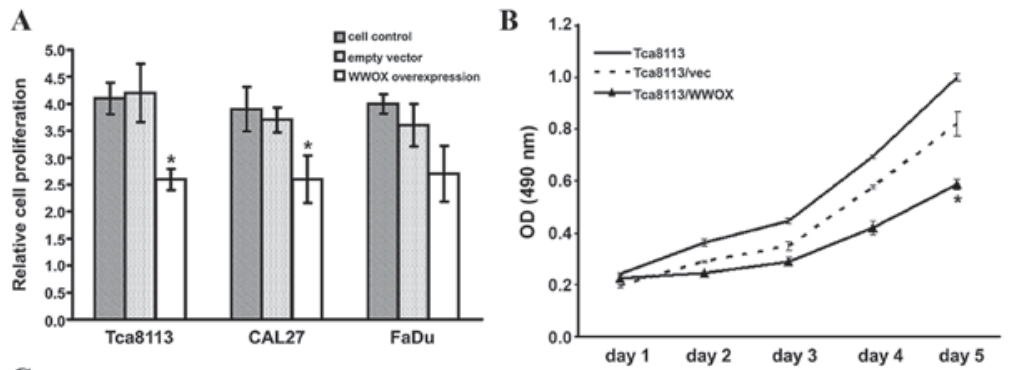

C
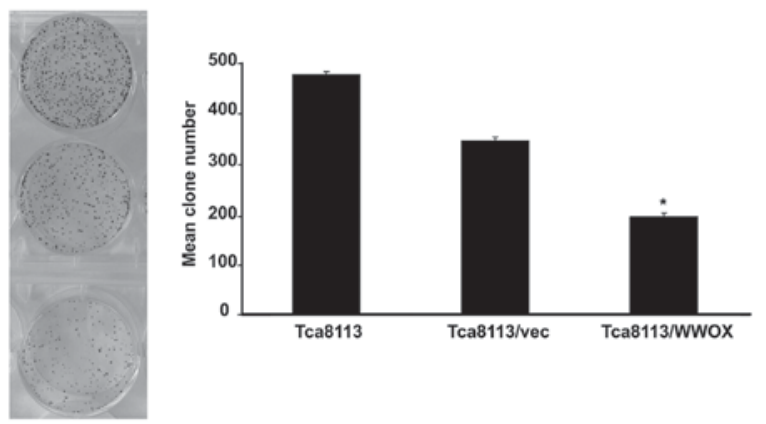

D
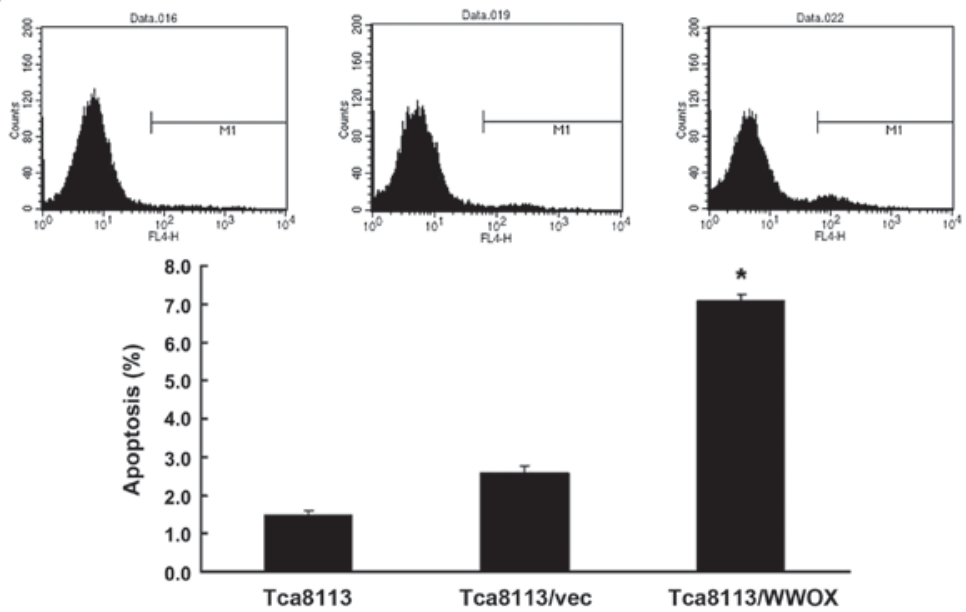

E
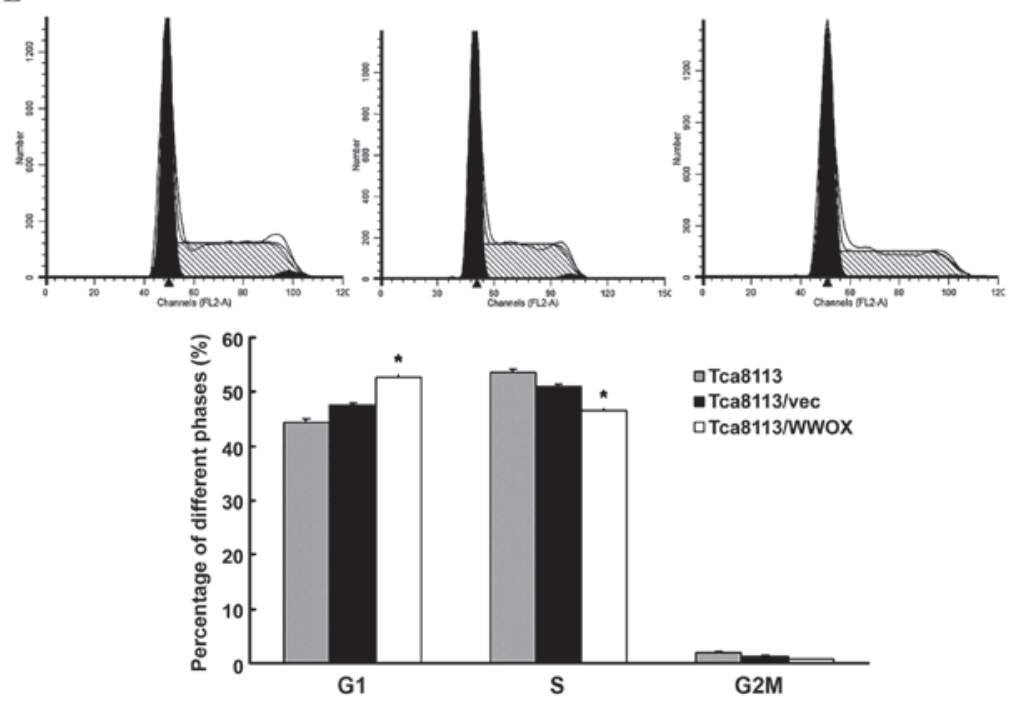

Figure 2. Effect of WWOX on Tca8113 cells in vitro. (A) Relative cell proliferation rate, demonstrating that WWOX overexpression significantly inhibited the growth of three types of oral squamous carcinoma cells, as determined by MTT assay. " $\mathrm{P}<0.05$ compared with the Tca8113/vec group. (B) Cell proliferation rates of Tca8113 cells with or without WWOX overexpression. ${ }^{*} \mathrm{P}<0.05$ compared with the Tca8113/vec group. (C) Colony formation assay, showing a reduced colony-forming ability in Tca8113 cells with WWOX overexpression. " $\mathrm{P}<0.05$ compared with the Tca8113/vec group. (D) Apoptosis analysis, demonstrating that the apoptosis rate of the Tca8113 cells with forced WWOX expression was high, as assessed by flow cytometry. "P<0.05 compared with the Tca8113/vec group. (E) Cell cycle analysis, demonstrating that WWOX overexpression yielded an increased level of G0/G1 arrest in the Tca8113 cells. All data are presented as the mean \pm standard deviation of three independent experiments. "P<0.05 compared with the Tca8113/vec group. WWOX, WW domain-containing oxidoreductase; OD, optical density; vec, empty vector. 

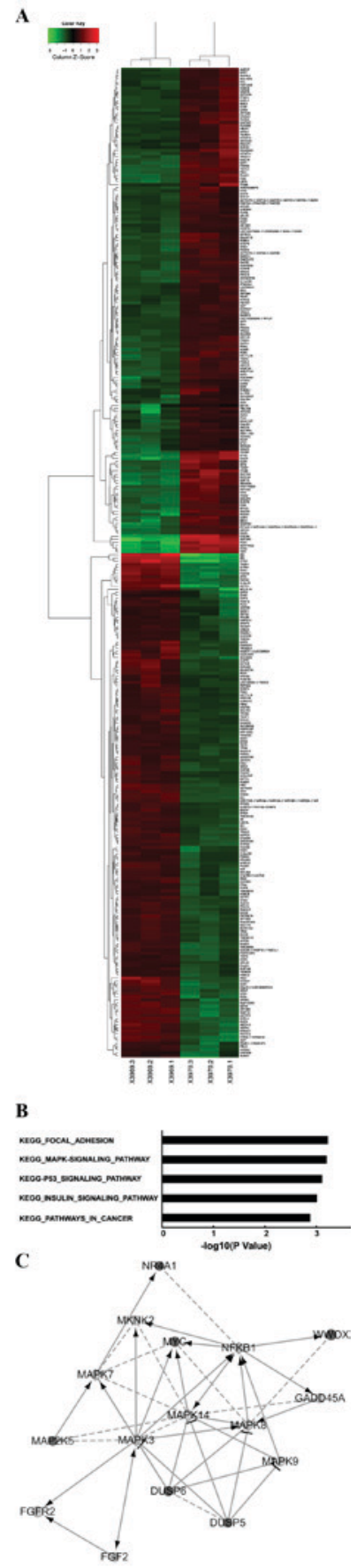

Figure 3. Global changes in the Tca8113 cell transcriptome with WWOX overexpression. (A) Heat map depicting 347 transcripts, which were significantly differentially expressed in Tca8113 cells with WWOX overexpression $(\mathrm{P}<0.05)$. The rows and columns represent samples and transcriptomes, respectively. The red and green colors represent upregulated and downregulated genes, respectively. X3969.1-X3969.3 represents the samples from the Tca8113 cells carrying empty vectors and X3970.1-X3970.3 represent the samples from the Tca8113 cells overexpressing the WWOX gene. (B) Functional pathway analysis of the differentially expressed genes was conducted by Reactome Functional Interaction network. In the Tca 8113 cells with WWOX overexpression, the five pathways modulated significantly by WWOX overexpression are depicted, indicated by the inverse logarithm of P-values. (C) Network between the WWOX gene and the MAPK signaling pathway. In the map, black circles, grey circles and grey squares are used to indicate changes in gene expression: Upregulation, downregulation and predicted genes, respectively. The larger the diameter of the circle is, the more significant the difference between the two groups. Solid lines indicate confirmed interactions and dashed lines represent predicted interactions. WWOX, WW domain-containing oxidoreductase; MAPK, mitogen-activated protein kinase; vec, empty vector; KEGG, Kyoto Encyclopedia of Genes and Genomes.

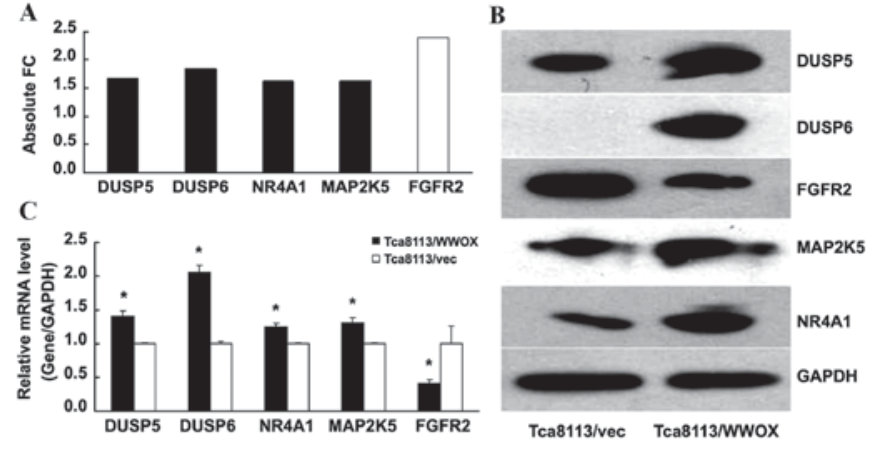

Figure 4. WWOX modulates the expression of genes involved in mediating MAPK signaling. (A) Absolute FC indicates the expression level of genes, compared with that of the Tca8113/vec control (which was equal to 1). Black columns represent upregulated genes and white columns represent downregulated genes. (B) Western blot analysis of the proteins was conducted, including the cells with and without WWOX overexpression. (C) The relative mRNA level of DUSP5, DUSP6, NR4A1, MAP2K5 and FGFR2 was detected by reverse transcription-quantitative polymerase chain reaction, normalized to GAPDH and compared with the control (which was equal to 1 ). "P<0.05 compared with the Tca8113/vec cells. WWOX, WW domain-containing oxidoreductase; DUSP, dual-specificity phosphatase; NR4A1, nuclear receptor subfamily 4 group A member 1; MAP2K5, mitogen-activated protein kinase kinase 5; FGFR2, fibroblast growth factor receptor 2; FC, fold-change; vec, empty vector; mRNA, messenger RNA.

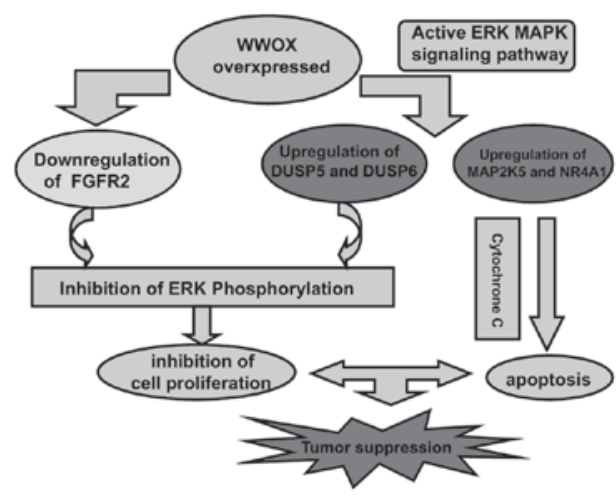

Figure 5. The proposed tumor-suppressor mechanism for the WWOX gene. WWOX, WW domain-containing oxidoreductase; DUSP, dual-specificity phosphatase; NR4A1, nuclear receptor subfamily 4 group A member 1; MAP2K5, mitogen-activated protein kinase kinase 5; FGFR2, fibroblast growth factor receptor 2; ERK, extracellular-signal regulated protein kinase; MAPK, mitogen-activated protein kinase.

the genes were examined by RT-qPCR and western blotting, yielding results that were for the majority of genes consistent with the initial findings of the present study $(\mathrm{P}<0.05$; Fig. 4B and C), indicating the upregulation of DUSP5, DUSP6, NR4A1 and MAP2K5, and the downregulation of FGFR2, with respect to mRNA and protein levels.

\section{Discussion}

WWOX has been identified as a gene located on a chromosomal fragile site, and deficiency/deletion with respect to the expression of the gene is common in various types of carcinoma (6). Reduced expression was also demonstrated in OSCC and oral precancerous lesions subsequent to examination with PCR and immunohistochemistry (8-10). Reduced 
expression of WWOX contributed to tumor development and progression, and exogenous WWOX expression significantly suppressed tumor growth $(13,14)$. Qu et al $(15)$ revealed that the reconstitution of WWOX inhibited cell proliferation and induced apoptosis, while the knockdown of WWOX resulted in the opposite effect in cervical cancer cells. Lin et al (16) concluded that WWOX suppressed prostate cancer cell progression by inducing cell cycle arrest in the G1 phase. The present study investigated the effect of WWOX overexpression on cell growth in oral squamous carcinoma cells, and the results are consistent with the findings of previous studies with respect to the WWOX gene inhibiting cell proliferation, and promoting apoptosis and cell cycle arrest. To investigate the underlying tumor-suppression mechanism of the WWOX gene, the present study used microarray analysis to evaluate the genetic changes exhibited in Tca8113 cells subsequent to WWOX overexpression.

To investigate the underlying tumor-suppression mechanism of the WWOX gene, the present study analyzed the genetic change of Tca8113 cells following WWOX overexpression by microarray analysis, and noticed an increase in the expression of DUSP5, DUSP6, NR4A1 and MAP2K5, and a decrease in the expression of FGFR2. These genes are closely associated with the extracellular-signal regulated protein kinase (ERK)/MAPK signaling pathway, and mediate various biological events involved in cell proliferation, differentiation and survival (17).

DUSP5 and DUSP6 are members of the MAPK phosphatase family (18). Okudela et al (19), Li et al (20) and Nunes-Xavier et al (21) observed that DUSP5 and DUSP6 act as negative mediators in the regulation of ERK1/2 phosphorylation and cell growth in tumor cells. Wang et al (18) indicated that, in corneal epithelial cells, DUSP6 overexpression specifically prevented the formation of phosphorylated ERK1/2 and slowed cell growth, whereas DUSP5 knockdown was observed to enhance ERK1/2 phosphorylation and cell growth. The authors therefore concluded that DUSP5 and DUSP6 serve a role in the negative feedback regulation of ERK/MAPK signaling when their expression is upregulated through the activation of the ERK/MAPK signaling pathway. The present study demonstrated that, subsequent to WWOX overexpression, the increased expression of DUSP5 and DUSP6 is accompanied by the inhibition of Tca8113 cell growth. Therefore, the present study hypothesizes that WWOX overexpression activates the ERK/MAPK signaling pathway, and upregulates the expression of DUSP5 and DUSP6. Conversely, DUSP5 and DUSP6 reduce ERK phosphorylation, and suppress the growth of Tca8113 cells.

NR4A1, also referred to as Nur77, is a member of the nuclear receptor subfamily 4 , group $\mathrm{A}$, and can be activated via a cascade involving MAP2K5, MAPK7 and NR4A1, which is also dependent on the ERK/MAPK signaling pathway (22). In OSCC, NR4A1 activated through the MAPK signaling pathway can induce apoptosis (23). The present study demonstrated that the combination of the upregulation of NR4A1 and MAP2K5 increased the level of apoptosis subsequent to WWOX overexpression in Tca8113 cells. Previous studies identified that NR4A1 induces apoptosis by associating with B-cell lymphoma 2 and initiating the release of cytochrome $c(23,24)$. Zhang et al $(25)$ reported that the ectopic overexpression of WWOX also induces a release of cytochrome $c$ from the mitochondria. As a result, the present study hypothesizes that the overexpression of WWOX upregulates the expression of MAP2K5 and NR4A1 by activating the ERK/MAPK signaling pathway, and induces apoptosis in Tca8113 cells through the release of cytochrome $c$.

FGFR2 is a tyrosine kinase receptor that is crucial with respect to controlling tumor proliferation, angiogenesis, migration and survival (26). Katoh and Nakagama (27) demonstrated that the expression of FGFR2 was amplified in breast and gastric cancer. In the colorectal cancer NCI-H716 cell line, which exhibits a high expression of FGFR2, the inhibition of FGFR2 by small molecule inhibitors or FGFR2 short hairpin (sh)RNA was shown to decrease cell viability (28). In pancreatic cancer, tumor cells with FGFR2-shRNA transfection exhibited attenuated proliferation rates, migration and invasion levels, and a reduced level of phosphorylation of ERK compared with that of the control cells (29). These findings demonstrate that the inhibition of FGFR2 contributes to the suppression of cell proliferation and ERK phosphorylation. In the present study, a reduced expression of FGFR2 and the inhibition of growth in Tca8113 cells were also observed when WWOX was overexpressed.

In summary, the overexpression of the WWOX gene in Tca8113 cells suppressed cell growth, and induced apoptosis and cell cycle arrest. This tumor suppression is associated with a modulation of the expression of genes that mediate the ERK/MAPK signaling pathway. The conclusions of the present study regarding the tumor-suppressor functions of the WWOX gene in a diagrammatic sketch are presented in Fig. 5. Targeting WWOX may be an effective method for the treatment of oral cancer.

\section{Acknowledgements}

The present study was supported by grants from the Public Welfare Technology and Application Research Projects (grant no. 2013C37019), the Science and Technology Plans of Taizhou City (grant no. 131ky17) and the Science and Technology Development Plan of Jilin City (grant no. 20163066).

\section{References}

1. Minicucci EM, da Silva GN and Salvadori DM: Relationship between head and neck cancer therapy and some genetic endpoints. World J Clin Oncol 5: 93-102, 2014.

2. Ratajczak-Wrona W, Jablonska E, Antonowicz B, Dziemianczyk D and Grabowska SZ: Levels of biological markers of nitric oxide in serum of patients with squamous cell carcinoma of the oral cavity. Int J Oral Sci 5: 141-145, 2013.

3. Rivera $\mathrm{C}$ and Venegas B: Histological and molecular aspects of oral squamous cell carcinoma (Review). Oncol Lett 8: 7-11, 2014.

4. Warnakulasuriya S: Living with oral cancer: Epidemiology with particular reference to prevalence and life-style changes that influence survival. Oral Oncol 46: 407-410, 2010.

5. Giannola LI, De Caro V, Giandalia G, Siragusa MG, Paderni C, Campisi G and Florena AM: 5-Fluorouracil buccal tablets for locoregional chemotherapy of oral squamous cell carcinoma: Formulation, drug release and histological effects on reconstituted human oral epithelium and porcine buccal mucosa. Curr Drug Deliv 7: 109-117, 2010.

6. Lewandowska U, Zelazowski M, Seta K, Byczewska M, Pluciennik E and Bednarek AK: WWOX, the tumour suppressor gene affected in multiple cancers. J Physiol Pharmacol 60 (Supp 1): S47-S56, 2009 
7. Ekizoglu S, Muslumanoglu M, Dalay N and Buyru N: Genetic alterations of the WWOX gene in breast cancer. Med Oncol 29: $1529-1535,2012$.

8. Pimenta FJ, Cordeiro GT, Pimenta LG, Viana MB, Lopes J, Gomez MV, Aldaz CM, De-Marco L and Gomez RS: Molecular alterations in the tumor suppressor gene WWOX in oral leukoplakias. Oral Oncol 44: 753-758, 2008.

9. Pimenta FJ, Gomes DA, Perdigão PF, Barbosa AA, Romano-Silva MA, Gomez MV, Aldaz CM, De Marco L and Gomez RS: Characterization of the tumor suppressor gene WWOX in primary human oral squamous cell carcinomas. Int J Cance 118: 1154-1158, 2006.

10. Pimenta FJ, Cordeiro GT, Pimenta LG, Viana MB, Lopes J, Gomez MV, Aldaz CM, De Marco L and Gomez RS: Molecular alterations in the tumor suppressor gene WWOX in oral leukoplakias. Oral Onco 44: 753-758, 2008.

11. Kabbout M, Garcia MM, Fujimoto J, Liu DD, Woods D, Chow CW, Mendoza G, Momin AA, James BP, Solis L, et al: ETS2 mediated tumor suppressive function and MET oncogene inhibition in human non-small cell lung cancer. Clin Cancer Res 19: 3383-3395, 2013.

12. Livak KJ and Schmittgen TD: Analysis of relative gene expression data using real-time quantitative PCR and the 2(-Delta Delta C(T)) Method. Methods 25: 402-408, 2001

13. Aqeilan RI, Abu-Remaileh M and Abu-Odeh M: The common fragile site FRA16D gene product WWOX: Roles in tumor suppression and genomic stability. Cell Mol Life Sci 71: $4589-4599,2014$

14. Zhang H, Kong L, Cui Z, Du W, He Y, Yang Z, Wang L and Chen X: The WWOX gene inhibits the growth of U266 multiple myeloma cells by triggering the intrinsic apoptotic pathway. Int J Mol Med 34: 804-809, 2014

15. Qu J, Lu W, Li B, Lu C and Wan X: WWOX induces apoptosis and inhibits proliferation in cervical cancer and cell lines. Int J Mol Med 31: 1139-1147, 2013.

16. Lin JT, Li HY, Chang NS, Lin CH, Chen YC and Lu PJ: WWOX suppresses prostate cancer cell progression through cyclin D1-mediated cell cycle arrest in the G1 phase. Cell Cycle 14: 408-416, 2015

17. Whelan JT, Hollis SE, Cha DS, Asch AS and Lee MH: Post-transcriptional regulation of the Ras-ERK/MAPK signaling pathway. J Cell Physiol 227: 1235-1241, 2012.

18. Wang Z, Reinach PS, Zhang F, Vellonen KS, Urtti A, Turner H and Wolosin JM: DUSP5 and DUSP6 modulate corneal epithelial cell proliferation. Mol Vis 16: 1696-1704, 2010.
19. Okudela K, Yazawa T, Woo T, Sakaeda M, Ishii J, Mitsui H, Shimoyamada $\mathrm{H}$, Sato $\mathrm{H}$, Tajiri $\mathrm{M}$, Ogawa $\mathrm{N}$, et al: Down-regulation of DUSP6 expression in lung cancer: Its mechanism and potential role in carcinogenesis. Am J Pathol 175: 867-881, 2009.

20. Li W, Song L, Ritchie AM and Melton DW: Increased levels of DUSP6 phosphatase stimulate tumourigenesis in a molecularly distinct melanoma subtype. Pigment Cell Melanoma Res 25: 188-199, 2012.

21. Nunes-Xavier CE, Tárrega C, Cejudo-Marín R, Frijhoff J, Sandin A, Ostman A and Pulido R: Differential up-regulation of MAP kinase phosphatases MKP3/DUSP6 and DUSP5 by Ets2 and c-Jun converge in the control of the growth arrest versus proliferation response of MCF-7 breast cancer cells to phorbol ester. J Biol Chem 285: 26417-26430, 2010.

22. Mori Sequeiros Garcia M, Gorostizaga A, Brion L, González-Calvar SI and Paz C: cAMP-activated Nr4a1 expression requires ERK activity and is modulated by MAPK phosphatase-1 in MA-10 Leydig cells. Mol Cell Endocrinol 408: 45-52, 2015

23. Liu PY, Sheu JJ, Lin PC, Lin CT, Liu YJ, Ho LI, Chang LF, Wu WC, Chen SR, Chen J, et al: Expression of Nur77 induced by an $n$-butylidenephthalide derivative promotes apoptosis and inhibits cell growth in oral squamous cell carcinoma. Invest New Drugs 30: 79-89, 2012.

24. Thompson J and Winoto A: During negative selection, Nur77 family proteins translocate to mitochondria where they associate with Bcl-2 and expose its proapoptotic BH3 domain. J Exp Med 205: 1029-1036, 2008.

25. Zhang P, Jia R, Ying L, Liu B, Qian G, Fan X and Ge S: WWOX-mediated apoptosis in A549 cells mainly involves the mitochondrial pathway. Mol Med Rep 6: 121-124, 2012.

26. Feng S, Zhou L, Nice EC and Huang C: Fibroblast growth factor receptors: Multifactorial-contributors to tumor initiation and progression. Histol Histopathol 30: 13-31, 2015.

27. Katoh $\mathrm{M}$ and Nakagama H: FGF receptors: Cancer biology and therapeutics. Med Res Rev 34: 280-300, 2014.

28. Mathur A, Ware C, Davis L, Gazdar A, Pan BS and Lutterbach B: FGFR2 is amplified in the NCI-H716 colorectal cancer cell line and is required for growth and survival. PLoS One 9: e98515, 2014.

29. Matsuda Y, Yoshimura H, Suzuki T, Uchida E, Naito Z and Ishiwata T: Inhibition of fibroblast growth factor receptor 2 attenuates proliferation and invasion of pancreatic cancer. Cancer Sci 105: 1212-1219, 2014. 